\title{
A Dynamic Deep Sleep Stage in Drosophila
}

\author{
Bart van Alphen, Melvyn H.W. Yap, ${ }^{*}$ Leonie Kirszenblat, ${ }^{*}$ Benjamin Kottler, and Bruno van Swinderen \\ Queensland Brain Institute, The University of Queensland, St Lucia, Queensland 4072, Australia
}

How might one determine whether simple animals such as flies sleep in stages? Sleep in mammals is a dynamic process involving different stages of sleep intensity, and these are typically associated with measurable changes in brain activity (Blake and Gerard, 1937; Rechtschaffen and Kales, 1968; Webb and Agnew, 1971). Evidence for different sleep stages in invertebrates remains elusive, even though it has been well established that many invertebrate species require sleep (Campbell and Tobler, 1984; Hendricks et al., 2000; Shaw et al., 2000; Sauer et al., 2003). Here we used electrophysiology and arousal-testing paradigms to show that the fruit fly, Drosophila melanogaster, transitions between deeper and lighter sleep within extended bouts of inactivity, with deeper sleep intensities after $\sim 15$ and $\sim 30$ min of inactivity. As in mammals, the timing and intensity of these dynamic sleep processes in flies is homeostatically regulated and modulated by behavioral experience. Two molecules linked to synaptic plasticity regulate the intensity of the first deep sleep stage. Optogenetic upregulation of cyclic adenosine monophosphate during the day increases sleep intensity at night, whereas loss of function of a molecule involved in synaptic pruning, the fragile-X mental retardation protein, increases sleep intensity during the day. Our results show that sleep is not homogenous in insects, and suggest that waking behavior and the associated synaptic plasticity mechanisms determine the timing and intensity of deep sleep stages in Drosophila.

\section{Introduction}

Most animals endowed with a brain require daily sleep (Campbell and Tobler, 1984; Cirelli and Tononi, 2008), and this state of behavioral quiescence is thought to be crucial for a number of processes related to cognition (Killgore, 2010). Sleep is often first identified in animals by prolonged immobility, but is better characterized by decreased responsiveness to stimuli (Campbell and Tobler, 1984). Other criteria, such as homeostatic regulation, neural correlates in brain activity, and modulation by stimulants such as caffeine are also typically used to measure sleep physiology in animals (van Swinderen, 2006). In humans, sleep is a dynamic process in which the brain transitions through different stages of activity such as the rapid eye movement (REM) stage and three non-REM stages, including slow-wave sleep (SWS). These distinct stages, typically identified by electroencephalographic recordings (Rechtschaffen and Kales, 1968), are associated with different arousal thresholds. In humans, lower levels of behavioral responsiveness to stimuli occur during SWS and comparatively higher responsiveness levels occur during REM sleep (Rechtschaffen et al., 1966). Whereas both REM sleep and

Received Jan. 7, 2013; revised March 9, 2013; accepted March 11, 2013.

Author contributions: B.v.A., M.H.W.Y., L.K., B.K., and B.v.S. designed research; B.v.A., M.H.W.Y., and L.K. performed research; B.K. contributed unpublished reagents/analytic tools; B.v.A., M.H.W.Y., L.K., and B.v.S. analyzed data; B.v.A. and B.v.S. wrote the paper.

This work was supported by the Australian Research Council (Discovery Project Grant \#DP1093968 and Future Fellowship \#FT100100725 to B.v.S.). We thank Martin Schwaerzel for generously providing the UAS-PAC $\alpha$ strain, Paul Shaw for discussions on the manuscript, Angelique Paulk for brain dissections and artwork, and Yan-Qiong Zhou for help with the fly-ball design.

*M.H.W.Y and L.K. contributed equally to this paper.

Correspondence should be addressed to Bruno van Swinderen, Queensland Brain Institute, The University of Queensland, Building \#79, Brisbane, QLD, 4072 Australia. E-mail: b.vanswinderen@uq.edu.au.

DOI:10.1523/JNEUROSCI.0061-13.2013

Copyright $\odot 2013$ the authors $\quad 0270-6474 / 13 / 336917-11 \$ 15.00 / 0$
SWS have been proposed to play an important role in memory consolidation (Diekelmann and Born, 2010), SWS has been proposed to be specifically involved in maintaining synaptic homeostasis in mammals (Tononi and Cirelli, 2003, 2006).

Recent research on sleep genetics in model organisms, such as the fruit fly Drosophila melanogaster, has provided evidence that sleep processes engage molecular mechanisms that regulate synaptic function (Bushey et al., 2009; Donlea et al., 2009; Gilestro et al., 2009; Bushey et al., 2011), although flies show no evidence of the slow-wave brain activity associated with deep sleep in mammals (Nitz et al., 2002). Although oscillatory brain activity has been found in sleeping crayfish (Ramón et al., 2004) and honeybees and cockroaches appear to display varying levels of sleep intensity (Tobler and Neuner-Jehle, 1992; Sauer et al., 2003; Eban-Rothschild and Bloch, 2008), sleep/wake in Drosophila has been conventionally quantified as a bimodal process. Based on early sleep research in this model, the duration of fly inactivity reflects accrued sleep processes (Andretic and Shaw, 2005) and a fly is considered to be asleep if it has been inactive for $5 \mathrm{~min}$ or more (Shaw et al., 2000; Huber et al., 2004). Although the 5 min inactivity threshold has been invaluable for understanding sleep processes in this model, and has already provided insight on sleep intensity in flies (Huber et al., 2004), it remains unclear whether flies, like mammals, display defined sleep stages that might be associated with specific cellular functions. This question has become increasingly relevant since Drosophila became a model of choice for investigating molecular processes associated with sleep in health and disease (van Alphen and van Swinderen, 2013). In this study, we investigated whether flies display changing levels of sleep intensity throughout the day and night and within extended sleep bouts. We then used behavioral and genetic manipulations to measure whether our sleep intensity metrics were affected in predictable ways. 


\section{Materials and Methods}

\section{Fly stocks and rearing conditions}

Flies were raised at $22^{\circ} \mathrm{C}$ on standard yeast-based Drosophila medium with a $12 \mathrm{~h}$ light-dark (LD) rhythm. The day before each experiment, adult Drosophila (4-8 d old) were briefly ( $<5 \mathrm{~min}$ ) anesthetized using $\mathrm{CO}_{2}$, after which males and females were transferred to plastic vials containing standard fly medium. Each vial contained $\sim 40-50$ male or female flies. The next day, individual flies were aspirated into $65 \mathrm{~mm}$ glass tubes (Trikinetics) containing fly medium on one end and sealed with a cotton plug on the other end. Canton-S (CS) and white ${ }^{1118}\left(\mathrm{w}^{1118}\right)$ are standard laboratory strains of fruit fly. The following lines were ordered from the Bloomington Stock Center: Fmr1 ${ }^{\Delta 50 M}$ (stock \#6930, w ${ }^{1118}$; Fmr1 ${ }^{\Delta 50 \mathrm{M}} / \mathrm{TM} 6 \mathrm{~B} ; \mathrm{Tb}^{1}$ ); elav-Gal4/Cyo; TM2/TM6B. UAS-PAC $\alpha$ flies were a gift from Martin Schwärzel (Schröder-Lang et al., 2007) and were crossed to elav-Gal4/Cyo; TM2/TM6B.

\section{Electrophysiology}

Brain recordings were performed as described previously (Nitz et al., 2002). Briefly, local field potentials (LFPs) were sampled at $300 \mathrm{~Hz}$ as a voltage differential from two glass electrodes inserted into the fly brain, one in each hemisphere. Electrode recording locations were verified by iontophoresis of Texas Red dye and subsequent brain imaging using standard brain dissection techniques and fluorescence microscopy. These were typically deep into either optic lobe, as described previously (van Swinderen, 2012). To monitor fly activity and rest, the tethered fly (only wild-type females were used for electrophysiology) was positioned on a humidified air-suspended ball upon which it could walk or rest at will (Fig. 1A). All flies used for electrophysiology $(n=13)$ survived at least $24 \mathrm{~h}$. Some flies were tethered in the morning and others in the evening to ensure that day and night effects were not purely a consequence of time on the tether. Filming of the preparation with a webcam (Logitech) at 3 frames/s under infrared light allowed for quantification of fly activity levels. For each frame, a pixelated, logical (black and white) picture was generated that could be subtracted from the preceding frame to generate a difference image ( $\Delta$ pixels) through time. The threshold for immobility was determined offline by visually comparing the activity trace (Fig. $1 B$ ) with the recorded video of the fly to determine the average number of pixels that changed from frame to frame for a completely inactive fly. This noise is caused by small fluctuations in lighting conditions and camera noise. A threshold was chosen that was +2 SD above the inactivity average for the whole trace ( 20 or 25 pixels for all flies; Fig. $1 E, F$, red line). Pixel fluctuations above this threshold were considered to be fly activity and flies were classified as being awake as soon as their activity was above their individual threshold, even for just one frame. Sleep duration was thus counted from the last instance of any activity (this was the sleep initiation point) plus $5 \mathrm{~min}$ or more of inactivity. LFP data were time stamped to match movement data. Fourier analyses of LFPs were performed using MATLAB software (MathWorks). For the summed frequency analysis, the $45-55 \mathrm{~Hz}$ range was removed to prevent line noise contamination. To quantify LFP dynamics within sleep bouts for daytime and nighttime sleep, we selected sleep bouts of 11-20 min because such data presumably included the 5 min sleep "transition" period. In addition, these are the longest sleep bouts that occur regularly (3-5 times per fly; Fig. 1D) during both day and night. For every minute of these sleep bouts, a Fourier transform was performed on the LFP data to determine its power spectrum. Low frequencies $(<10 \mathrm{~Hz})$ were removed to eliminate fluctuations caused by heart rate or respiratory activity. The resulting power spectrum was summed over the 11-40 and $41-80 \mathrm{~Hz}$ ranges. The lower frequencies $(11-40 \mathrm{~Hz})$ bracket a range in which we have previously observed attention-like effects in the LFP (van Swinderen and Greenspan, 2003). We contrast these effects to $41-80 \mathrm{~Hz}$ because these represent an adjoining range of frequencies in which we have not observed attention-like effects in the LFP.

\section{Behavioral assays}

Behavioral responsiveness to a mechanical stimulus. Flies were individually housed in $65 \mathrm{~mm}$ glass tubes (Trikinetics), 17 tubes on a tray, two trays per filmed experiment. Arousal thresholds were measured by subjecting flies to a mechanical stimulus using shaftless vibrating motors (model

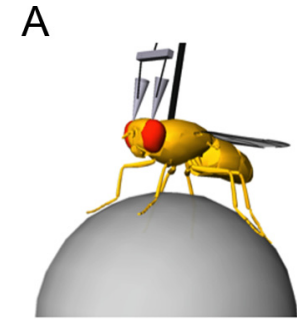

B
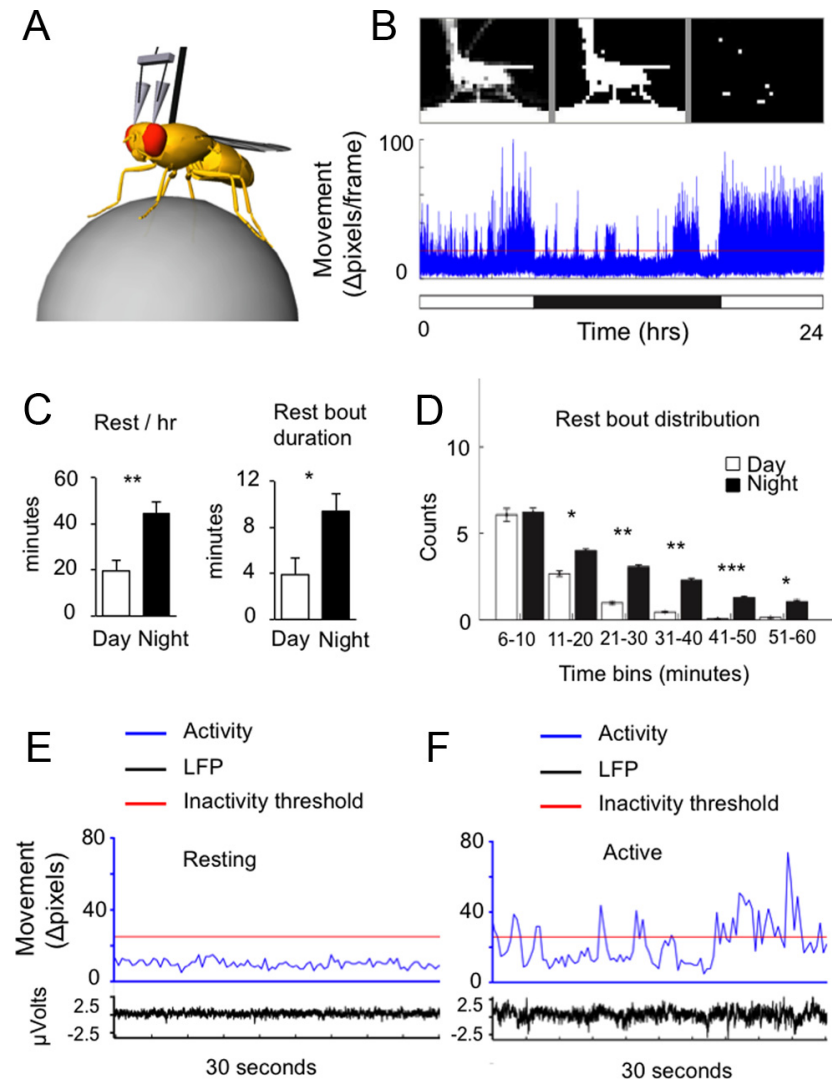

Figure 1. Recording brain activity in behaving flies. $A$, Brain-recording setup. $B$, Fly movement is quantified from a filmed experiment. Top: A pixel subtraction method defines fly movement. Bottom: Activity pattern for a sample fly during the day (white bar) and night (black bar). Movement scale is $\Delta$ pixels/frame. Threshold for inactivity is indicated by the red line. C, Left: Average minutes rest per hour ( \pm SEM) for tethered female flies on an air-suspended ball (prepared for electrophysiology), for day (white) and night (black). $n=13$ wild-type females. ${ }^{* *} p<0.01$ by $t$ test comparison of means. Right: Average rest bout length ( \pm SEM) for the same flie. ${ }^{*} p<0.05$ by $t$ test comparison of means. $\boldsymbol{D}$, Rest bout distribution (average number of times each rest bout occurred per fly \pm SEM for day and night). Longer rest bouts are more common at night. ${ }^{* *} p<0.001 ;{ }^{* *} p<0.01 ;{ }^{*} p<0.05$ by $t$ test comparison of means. $\boldsymbol{E}, 30$ s sample of LFP activity in a fly that has been inactive for over 5 min (black), with corresponding movement channel (blue) below the empirically set threshold (red line; see Materials and Methods). $\boldsymbol{F}, 30$ s sample of LFP in an active fly (black), with corresponding movement traces (blue) above the empirically set threshold (red line).

312-101; Precision Microdrives). Stimulus intensity was controlled by randomly modulating the voltage used to drive the motors to deliver $5 \times$ $200 \mathrm{~ms}$ pulses ( 1 pulse/s) between 0 and $1.2 \mathrm{~g}$ (steps of $0.3 \mathrm{~g}$ ) using a custom MATLAB program interfacing with the analog output channels of a USB data acquisition device (1280 LS; Measurement Computing). The unit $g$ was used to express vibration amplitude $(1 \mathrm{~g}$ equals the gravitational force at the surface of the earth, $980 \mathrm{~cm} / \mathrm{s}^{2}$ ). The Precision Microdrives motors had a linear relationship between input voltage and vibration, where an increase of $0.5 \mathrm{~V}$ results in an increased vibration of $0.3 \mathrm{~g}$, with a maximum of $1.2 \mathrm{~g}$ at $2.5 \mathrm{~V}$. When flies are startled by mechanical stimulation, this triggers a locomotion response (Sawamura et al., 2008). Flies were recorded at 15 frames/s for $1 \mathrm{~min}$ before ("baseline") and 1 min after ("startle") the stimulus using a high-resolution camera (Grasshopper; Point Gray Research). Images were stored for offline analysis with custom MATLAB software. Stimulus-induced locomotion was calculated by subtracting the average velocity during the minute before stimulus onset from the average velocity during $1 \mathrm{~min}$ after stimulus delivery. Some flies did not respond even at the strongest intensity $(1.2 \mathrm{~g})$; these were given a $1.5 \mathrm{~g}$ score and labeled as nonresponding. Experiments were conducted between 1:00 P.M. and 5:00 P.M.. In a separate set of experiments, a maximal vibration intensity $(1.2 \mathrm{~g})$ was delivered every hour for $24 \mathrm{~h}$, and velocity changes were monitored for 
$3 \mathrm{~d}$ and nights. Circadian effects on arousal were tested by raising flies in LD conditions and then transferring them to the arousal-testing incubator for $3 \mathrm{~d}$, with lights turned permanently off (DD condition) or on (LL condition).

Arousal thresholds. Arousal thresholds were tested with sequentially increasing vibration intensities from 0 to $1.2 \mathrm{~g}$ in $0.3 \mathrm{~g}$ (200 ms) increments every $10 \mathrm{~s}$, once an hour for $24 \mathrm{~h}$. For these experiments, a slightly different protocol was used, based on visual observation of filmed experiments. Arousal thresholds were calculated by assigning the $g$ that evoked a locomotion response (walking at least half the length of the glass tube) in quiescent animals (i.e., flies that had not shown any movement in the preceding minute), and determining the distribution of $g$ values for a strain. Because data were nonparametric, Kruskal-Wallis analyses were used to test for significant effects among strains or conditions.

Automated arousal testing over $72 \mathrm{~h}$. For sleep intensity experiments, two trays of 17 flies each were placed in an incubator at $22^{\circ} \mathrm{C}$ and a $12 \mathrm{~h} \mathrm{LD}$ cycle (lights on at 8:00 A.M.). Fly activity over $72 \mathrm{~h}$ was recorded using a webcam (Logitech 9000) modified to record infrared light (http://www. pysolo.net/docs/hacking-a-webcam-to-improve-lighting-conditions/). Visible light was filtered using an infrared long-pass filter (stock \#NT43-948; Edmund Optics). Images were taken with Pysolo (Gilestro and Cirelli, 2009) at 1 frame/s and stored for offline analysis using custom MATLAB software. Every hour, flies were subjected to a stimulus consisting of 5200 ms pulses at $1.2 \mathrm{~g}$. Responsiveness was determined for each fly at each time point using the velocity criteria described above. For each stimulus, percentages of responding flies were obtained by determining the fraction of flies that showed a significant increase in velocity using a twosample Kolgomorov-Smirnov test to determine whether the velocities during the minute before and after the startle stimulus were part of the same distribution. Because filming was continuous for these experiments, flies were grouped into different $1 \mathrm{~min}$ bins (from 0 to $59 \mathrm{~min}$ ) depending on how long they had been inactive before the test stimulus. Filmed analysis was also performed on some experiments to measure brief awakenings (BAs), as described previously (Huber et al., 2004), because in that study BAs were found to be correlated with sleep intensity. Filmed activity traces (velocity) were divided into 1 min bins and converted into binary data ( $1=$ active, $0=$ inactive $)$ based on our activity threshold. By searching for " 010 " sequences, we were able to calculate the average number of BAs/h. Our filmed analyses were consistent with previously published data for decreased BAs in sleep-deprived flies (Huber et al., 2004): BA/h: male day, ZT1-3, $1.13 \pm 0.20$; male day, sleepdeprived ZT1-3, $0.65 \pm 0.14$ ( $p=0.037)$; female day, ZT1-3, $0.98 \pm$ 0.15 ; female day, sleep-deprived ZT1-3, $0.53 \pm 0.20$ ( $p=0.045$, by $t$ test comparing means). In addition, there were differences in BAs between daytime and nighttime sleep, consistent with our finding that sleep intensity is different between day and night: $\mathrm{BA} / \mathrm{h}$ : male day, $1.01 \pm 0.18$; male night, $0.40 \pm 0.03$ ( $p=0.01)$; female day, $0.98 \pm 0.15$; female night, $0.53 \pm 0.20$ ( $p=0.001$, by $t$ test comparing means).

Fly activity. For some experiments, activity was measured using the infrared Drosophila Activity Monitor System (DAMS; Trikinetics). Because flies move back and forth in the tube, they interrupt an infrared beam at the center of the tube. These interruptions were counted, stored per minute, and analyzed offline using MATLAB. Fly sleep in these devices was defined as any period of inactivity longer than $5 \mathrm{~min}$, as described previously (Shaw et al., 2000; Huber et al., 2004). Average daytime sleep bout duration ( \pm SEM) for our wild-type strain was $27.7 \pm 1.9$ (males) and $21.4 \pm 1.8$ (females). Average night time sleep bout duration was $32 \pm 0.2$ (males) and $35 \pm 0.2$ (females) ( $n=32$ flies per sex).

Sleep deprivation. Individual flies in glass tubes were placed in a SNAP device (Shaw et al., 2002) and sleep deprived for $24 \mathrm{~h}$. One mechanical stimulus was delivered every $20 \mathrm{~s}$, which caused the flies to be tapped down their respective tubes. Sleep deprivation ran from 8:00 A.M. until 8:00 A.M. the next morning or from 8:00 P.M. until 8:00 P.M. the next evening, after which flies were transferred to the arousal setup to measure responsiveness during rebound sleep during the flies' subjective day or night. Sleep deprivation effects were also confirmed by identifying sleep rebound in the Trikinetics setup (see above).

Socialization. Adult CS flies (3-4 d old) were briefly anesthetized using $\mathrm{CO}_{2}$ and transferred to plastic vials (25 mm diameter, $100 \mathrm{~mm}$ length) containing food. The socialized groups consisted of 40-50 flies (males and females were kept separate). The isolated group consisted of a single fly in each vial. After $4 \mathrm{~d}$ of exposure to either a social or a socially impoverished environment, flies were transferred to the arousal testing setup.

\section{Image processing}

All image processing was done in MATLAB. After each experiment, fly positions were extracted from recorded images using an image subtraction method. For each recording session, a reference image was created by taking the average of all images recorded during that session. This averaging process eliminates all dynamic objects (the flies) and keeps all static objects (the rest of the setup). Fly positions were extracted by subtracting the reference image from each recorded image, transforming the resulting image to a logical black-and-white image, and calculating the center of mass for each fly. Fly position traces were differentiated to obtain velocity.

\section{Optogenetics}

PAC $\alpha$ activity was induced using two arrays of 32 blue LEDs (458 \pm 10 $\mathrm{nm}$, part \#FNL-U500B22WCSL; Element14). During arousal threshold experiments, which were conducted during the day in lit chambers, blue illumination started $10 \mathrm{~min}$ before the first trial and continued throughout the experiment. During $72 \mathrm{~h}$ arousal testing experiments, flies were illuminated with only blue light throughout the light period (8:00 A.M. to 8:00 P.M.) and this was turned off at night.

\section{Curve fitting}

To determine the point of deepest sleep intensity, third-order polynomials were fitted to the sleep intensity data, which had the following functional form:

$$
y=\mathrm{ax}^{\wedge} 3+\mathrm{bx}^{\wedge} 2+\mathrm{cx}+\mathrm{d}
$$

The first turning point of this equation corresponds to the moment when deepest sleep is achieved and can be determined by solving the polynomial's derivative for zero. The turning point that had the lowest value of $x$, which corresponds to the local minimum, was selected to determine the time and amplitude of deepest sleep.

To visualize sleep onset and intensity, responsiveness data for immobile flies was processed in four steps: (1) mean responsiveness data were normalized such that the responsiveness of active flies (at $0 \mathrm{~min}$ of inactivity) was set to a value of 1 ; (2) normalized data were smoothed using a 5-point moving average; (3) third-order polynomials were fitted to smoothed, normalized means; (4) the turning points in the curve were determined by solving the polynomial's derivative for zero:

$$
\mathrm{dy} / \mathrm{dx}=3^{\star} \mathrm{ax}^{\wedge} 2+2^{\star} \mathrm{bx}+\mathrm{c}=0
$$

\section{Statistical analyses}

All data were analyzed in MATLAB. Responsiveness to stimuli was analyzed using a two sample Kolgomorov-Smirnov test to determine whether the velocities during the minute before and after the startle stimulus were part of the same distribution. Arousal threshold data were nonparametric; therefore, Kruskal-Wallis analyses were used to test for significant effects among strains or conditions. Mean sleep durations were compared by the Wilcoxon rank-sum test. All data are presented as means \pm SEM. Comparisons of sleep intensity between strains and/or conditions was done by first fitting sleep intensity data ("fraction response") by a third-order polynomial (see above) and finding the deepest sleep point. A 5 min window was framed around this point and the original, non-smoothed data within this window were then averaged to determine sleep intensity at this point. These data were compared between strains and conditions to uncover significant differences in sleep intensity (by one-way ANOVA or Wilcoxon rank-sum test). Significance was set at $p<0.05$ for all statistical tests.

\section{Results \\ Electrophysiology}

To first investigate whether flies might display distinct sleep stages during prolonged bouts of inactivity, we performed brain 
recordings on individual animals (Fig. 1). In previous studies, we have shown that sleep in Drosophila is associated with overall decreased activity in the brain, as measured by LFPs (Nitz et al., 2002; van Swinderen et al., 2004), although LFP dynamics during sleep were not examined and sleep was defined according to criteria established for untethered flies ( $>5$ min of inactivity; Shaw et al., 2000; Huber et al., 2004). To record LFPs from behaving flies, we used a similar preparation as described previously (Nitz et al., 2002), with the difference that the tethered fly could walk (or rest) on an air-suspended ball during recordings (Fig. 1A). Filming of the fly under infrared lighting allowed movement to be quantified (see Materials and Methods) during the day and night (Fig. 1B). Filmed tracking of fly activity levels provided sufficient resolution for detecting when flies stopped moving entirely and for determining what happened in the brain LFP during prolonged inactivity (which is equivalent to immobility at our level of resolution; Fig. 1B). Tethered flies were significantly less active at night and the night was characterized by longer inactivity bouts than the day (Fig. 1C). However, tethered flies did regularly display extended bouts of inactivity ( $>5 \mathrm{~min}$ ) in this setup, day or night (Fig. $1 D$ ). We are assuming that this prolonged inactivity is a resting state.

After 5 min of inactivity, fly brain LFP activity became quite flat (Fig. 1E), as shown previously (Nitz et al., 2002). We are assuming that this is sleep. In contrast, the waking LFP was more active, even when the animal was not displaying any detectable movement for a few seconds (Fig. $1 F$ ). Analyses of LFPs confirmed that brain activity $(11-80 \mathrm{~Hz})$ in wild-type flies was significantly attenuated during sleep (Fig. 2A). However, daytime and nighttime sleep were not equivalent: the sleeping fly brain was significantly more active during the day than during the night (Fig. 2A). Wake LFP, in contrast, was not different between the day and the night (Fig. 2A). We next examined LFP activity through time during extended (11-20 min) sleep bouts (Fig. 2B). We found that LFP power $(11-80 \mathrm{~Hz}$ ) changed considerably during a sleep bout (Fig. $2 C, D$ ), although there was variability among flies in the timing of LFP changes in power. A closer examination of different LFP frequency domains (low versus high) revealed significant variability in the lower frequencies $(11-40 \mathrm{~Hz})$ depending on time since sleep initiation (Fig. 2E, minutes asleep), but there were no changes in higher frequencies during extended sleep bouts (Fig. $2 F$ ).

\section{Arousal thresholds}

Transiently floored $11-40 \mathrm{~Hz}$ activity during sleep (Fig. 2C-E) suggested a deeper sleep stage, because the average LFP power is already lower during sleep than wake (Fig. 2A). To determine whether these dynamic sleep processes were behaviorally relevant, we investigated whether flies displayed different levels of behavioral responsiveness during sleep. To test and measure behavioral responsiveness efficiently in flies, we designed an apparatus to deliver brief $(200 \mathrm{~ms})$ mechanical stimuli of varying (randomized) intensities ( $0-1.2 \mathrm{~g}$; see Materials and Methods) to groups of flies housed individually in small glass tubes (Fig. 3). Flies were filmed and their locomotor responses to the stimuli were tracked automatically (Fig. 3A). Startle responses increase with stimulus intensity in both males and females (Fig. $3 B, C$ ). By probing hourly for responses to robust vibration intensities (1.2 $g$ ), we were able to investigate how average behavioral responsiveness changed throughout several consecutive days and nights (Fig. 3D). As expected, responsiveness to the vibrations followed a robust day/night pattern, with decreased responsiveness at night and increased responsiveness during the day when flies
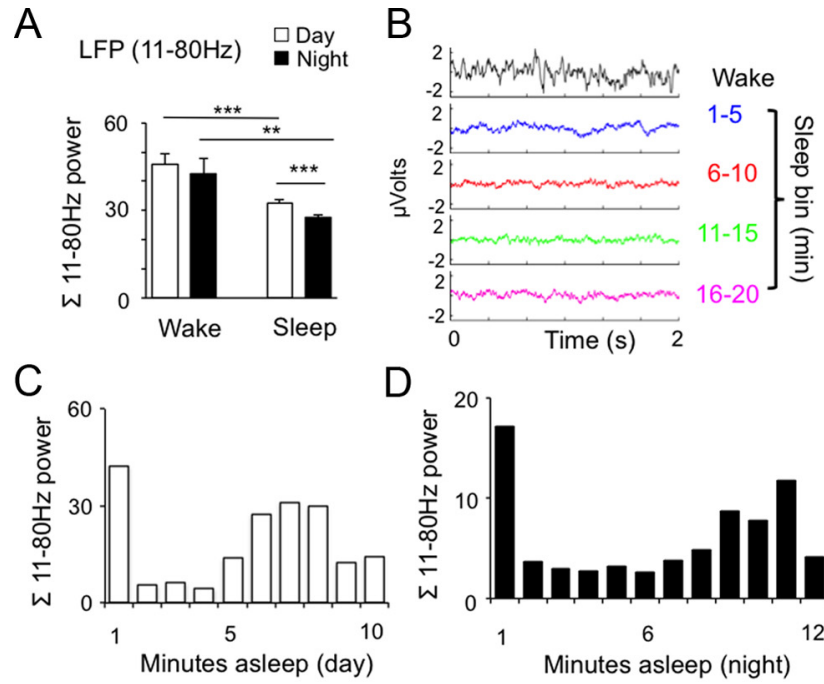

$\mathrm{D}$
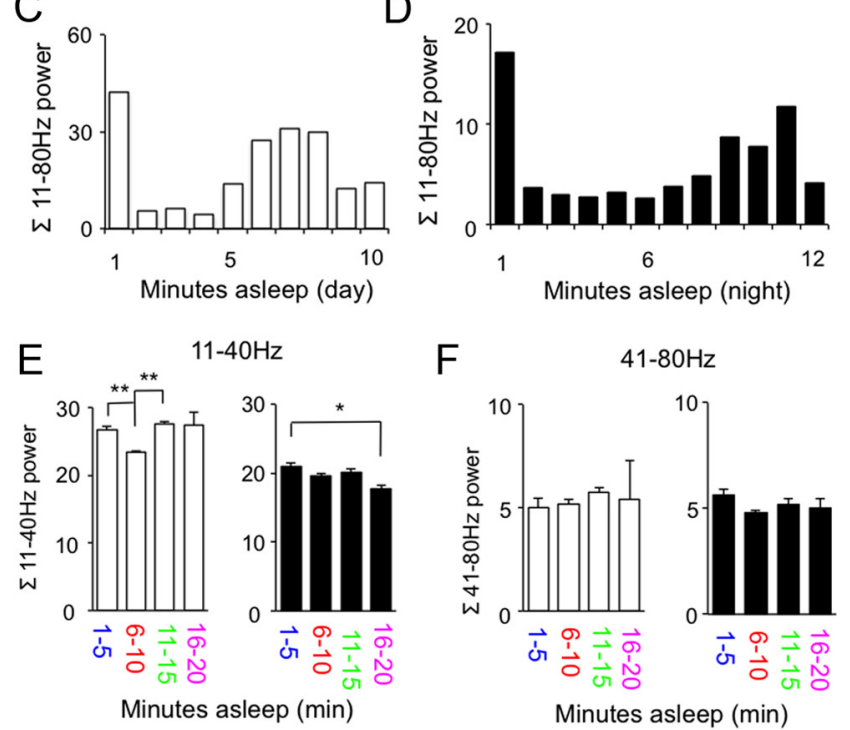

Figure 2. Changes in brain activity during fly sleep. $\boldsymbol{A}$, Waking LFP activity $\left(\sum 11-80 \mathrm{~Hz}\right.$ power \pm SEM) is significantly greater than sleeping LFP activity for day or night. Sleeping LFP activity is significantly greater during the day than during the night (all sleep epochs $>5 \mathrm{~min}$ were combined, $n=13$ female CS flies; ${ }^{* *} p<0.001,{ }^{* *} p<0.01$ by Wilcoxon rank-sum test. $\boldsymbol{B}$, Sample LFP activity taken at different times before and after initiation of a long sleep bout. Data are from the same fly taken from different 5 min epochs during one extended sleep bout (>20 min) at night. C, Summed $11-80 \mathrm{~Hz}$ LFP activity during a 10 min daytime sleep bout in a sample fly. $D$, Summed $11-80 \mathrm{~Hz}$ LFP activity during a 12 min nighttime sleep bout in a sample fly. $\boldsymbol{E}, \Sigma$ power ( \pm SEM) for $11-40 \mathrm{~Hz}$ LFP activity for consecutive $5 \mathrm{~min}$ bins after sleep initiation during the day (white) and the night (black). ${ }^{*} p<0.05$; ${ }^{* *} p<0.01$ by Wilcoxon rank-sum test. Time bins (minutes asleep) are color-coded as in $\boldsymbol{B}$. Only long (11-20 min) sleep bouts were analyzed. $\boldsymbol{F}, \Sigma$ power ( \pm SEM) for $41-80 \mathrm{~Hz}$ LFP activity for consecutive $5 \mathrm{~min}$ bins after sleep initiation during the day (white) and the night (black); same data as in $\boldsymbol{E}$. Time bins (minutes asleep) are color coded as in $\boldsymbol{B}$.

were exposed to $12 \mathrm{~h}$ LD cycles (Fig. $3 E-G$ ). Flies kept in DD also displayed increased responsiveness during their subjective day (Fig. $3 H$ ), but flies kept in LL displayed an intermediate level of behavioral responsiveness that was not different between day and night (Fig. 3I). Our startle paradigm thus accurately probed fly arousal under the LD conditions that are traditionally used to study sleep in Drosophila.

We therefore next examined arousal thresholds specifically in quiescent flies. By probing only nonmoving animals with increasing vibration intensities (Fig. $4 A$ ), we determined the minimal vibration intensity needed to evoke movement (arousal threshold; see Materials and Methods) in different individuals. By probing hourly over several days and nights, we then determined how arousal thresholds changed over $24 \mathrm{~h}$ (Fig. 4B). As expected, arousal thresholds were significantly lower during the day than at night in both males and females $(p<0.001,1 \mathrm{df}, \mathrm{H}=401.01$, Kruskal-Wallis test for medians, $n=60$, only male data are shown). Indeed, the arousal threshold profiles we found (Fig. 4B) closely resembled more conventional graphs plotting hourly 

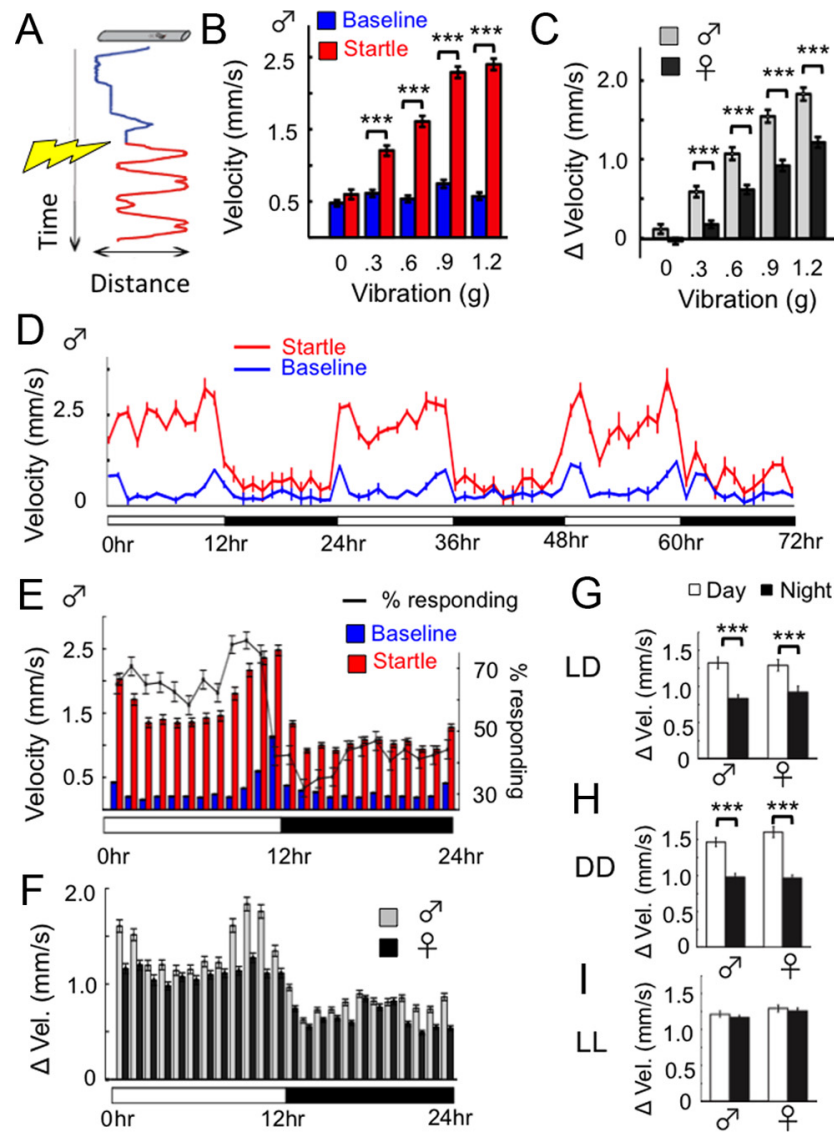

Figure 3. Stimulus-induced locomotion. $\boldsymbol{A}$, Detection of a startle stimulus (brief vibrations, indicated by yellow flash; see Materials and Methods) increases fly locomotor activity (blue, baseline locomotion; red, startle locomotion). $\boldsymbol{B}$, Startle responses (red) in wild-type males ( \pm SEM) increase with $g(n=136) .{ }^{* *} p<0.001$ by $t$ test compared with mean baseline velocity (blue). C, Stimulus-induced locomotion, the difference between startle and baseline responses, is higher in wild-type males than females. ${ }^{* * *} p<0.001$ by $t$ test of means. $D$, Sample experiment showing mean baseline velocity and startle responses to $1.2 \mathrm{~g}$ stimulus over $72 \mathrm{~h}$ for wild-type males ( $n=17$ ). $\boldsymbol{E}$, Baseline and stimulus-induced velocity ( \pm SEM) in response to hourly stimulation by $1.2 \mathrm{~g}$, for $24 \mathrm{~h}$ ( $n=136$ male wild-type flies). A close correspondence was found between stimulus-induced velocity and the percentage of flies responding (black line, \pm SEM; see Materials and Methods). F, Stimulus-induced locomotion ( \pm SEM) over $24 \mathrm{~h}$ for wild-type males and females ( $n=85$ per group). $\mathbf{G}-\boldsymbol{I}$, Average daytime and nighttime stimulus-induced locomotion for wild-type males and females in $12 \mathrm{~h} \mathrm{LD}$ cycles $(n=85)$ in DD after LD entrainment $(n=68)$, and in $\mathrm{LL}(n=51)$. Night and day are subjective for DD and LL based on entrainment schedules. ${ }^{* *} p<0.01 ;{ }^{* * *} p<0.001$ by $t$ test comparing means. Error bars show SEM for all graphs.

sleep duration in Drosophila based on a 5 min inactivity threshold (Hendricks et al., 2000; Shaw et al., 2000; Huber et al., 2004; Andretic and Shaw, 2005; Fig. 8E), validating our approach as a reliable indicator of arousal state in flies (see also Materials and Methods, where we describe "BAs" per hour, which was found to be a reliable indicator of sleep intensity in flies, as shown in an earlier study: Huber et al., 2004).

\section{Sleep intensity}

Sleep duration and sleep intensity are not necessarily correlated, as suggested by our brain-recording experiments. To investigate sleep intensity in our assay, we applied our arousal-testing paradigm to examine responsiveness levels during extended bouts of inactivity (as determined by video tracking) during the day or night. We measured behavioral responsiveness to a robust vibration stimulus $(1.2 \mathrm{~g})$ every hour for $3 \mathrm{~d}$, and then determined
A

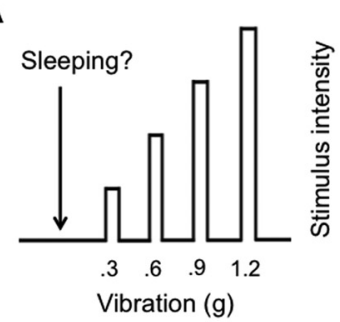

C

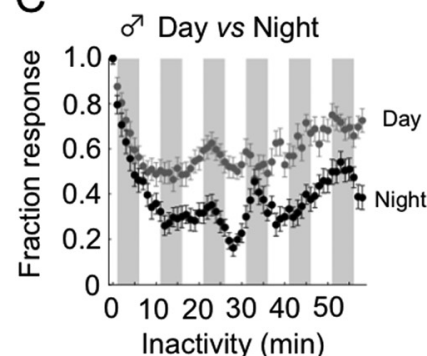

우 Day vs Night

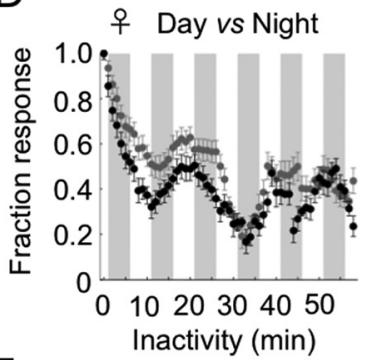

E

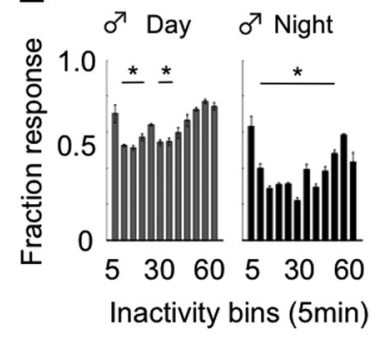

F
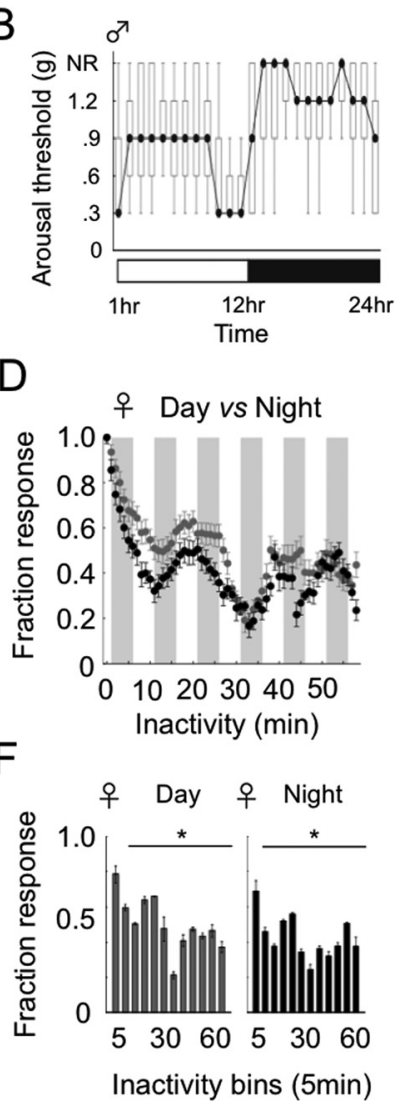

Figure 4. Different levels of sleep intensity in quiescent flies. $\boldsymbol{A}$, Increasing $g$ levels were used to determine the minimal level required to induce locomotion in an inactive fly. $\boldsymbol{B}$, Arousal thresholds (boxplots showing median g, black circles) $\pm 25-75$ th percentiles (gray boxes) and $\mathrm{min} /$ max values (whiskers) for wild-type males $(n=60$ ) across $24 \mathrm{~h}$. NR indicates nonresponding. $\boldsymbol{C}, \boldsymbol{D}$, Normalized response probability (fraction response) in wild-type males $(\boldsymbol{C}, n=85)$ and females $(\boldsymbol{D}, n=85)$ is plotted against minutes of inactivity before the stimulus (see Materials and Methods). A $1.2 \mathrm{~g}$ vibration stimulus was used to probe responsiveness every hour over several consecutive days. Gray circles indicate mean daytime responsiveness; black circles, mean nighttime responsiveness ( \pm SEM). Gray and white shading indicate 5 min inactivity bins. $\boldsymbol{E}, \boldsymbol{F}$, Data from $\boldsymbol{C}$ and $\boldsymbol{D}$ were binned into 5 min epochs. Mean responsiveness levels ( \pm SEM) were compared for different immobility durations within day (gray) or night (black) sleep bouts. *Bins that differed significantly $(p<0.05)$ from the first $(1-5 \mathrm{~min})$ bin by Tukey post hoc comparison.

whether the duration of prior immobility (only inactive flies were analyzed) predicted responsiveness probability. We compiled all ranges of inactivity from 1 to $59 \mathrm{~min}$ for day versus night (see Materials and Methods). We confirmed that nighttime sleep was on average deeper than daytime sleep, but also found that responsiveness probability varied depending on the time elapsed (Fig. $4 C, D)$. We confirmed that wild-type flies were significantly less responsive after 5 min of immobility (Shaw et al., 2000; Huber et al., 2004) and this was true in both males and females during the day and the night (Fig. $4 E, F$ ), but also that flies had already become significantly less responsive than moving flies after only 1 min of inactivity ( $p<0.01$ for males and females, day or night). Responsiveness decreased gradually in wild-type flies (i.e., sleep intensity increased) until a deeper sleep stage was achieved after $\sim 10$ min of immobility, for daytime or nighttime sleep in males and females (Fig. $4 C-F$ ). The timing of this deeper sleep stage is consistent with an early observation of a distinct postural change occurring at $14 \mathrm{~min}$ in a similar preparation (Hendricks et al., 2000). Interestingly, after this first period of deepest sleep intensity, responsiveness levels then appear to change again: sleep be- 
came lighter at $\sim 20 \mathrm{~min}$ and a second deep sleep stage was evident at $\sim 30 \mathrm{~min}$ in males and females, day or night, ending in comparatively lighter sleep as the quiescent hour proceeded (Fig. $4 C-F)$. Indeed, analysis of extended sleep bouts during the day revealed that male flies that had been quiescent for 45-60 min were as highly responsive as flies that had been quiescent for $<5$ min (Fig. 4E). Sleep intensity in flies thus depends on how long they have been asleep, but this relationship is nonlinear, with some evidence of what appears to be cycling behavior in arousability. Responsiveness differed significantly across the twelve 5 min bins (one-way ANOVA, males, day: $F_{(11,46)}=15.2, p<$ 0.001; males, night: $F_{(11,46)}=23.16, p<0.001$; females, day: $F_{(11,46)}=23.59, p<0.001$; females, night: $F_{(11,46)}=16.49$, $p<0.001)$.

Investigating sleep intensity stages by awakening animals with a stimulus, as we have done here, presents a fundamental conundrum: individual animals can only be awakened once during a sleep bout, after which they are obviously no longer asleep. So how can we investigate sleep dynamics in flies behaviorally? Our population assay suggests that, on average, flies are more deeply asleep after 10 and 30 min of immobility compared with other times, especially females (Fig. 4C,D). Although electrophysiology supported the view that sleep intensity levels may be dynamic throughout a sleep bout (Fig. 1, Fig. 2), it is nevertheless difficult to demonstrate behaviorally that this is happening in individual animals. To get around this problem, we investigated whether different groups of flies may be contributing disproportionally to different parts of the sleep intensity curves that we uncovered (Fig. $4 C, D$ ). In that experiment, we probed hourly for responsiveness and, for all animals that were immobile at the time of probing, we determined how long they had been immobile, thereby assigning them to an inactivity bin between 1 and $59 \mathrm{~min}$. We found that responsiveness levels were normally distributed among all inactive flies tested (Lilliefors test for normality, $p<$ 0.05; Fig. $5 A, B)$. Plotting the contributions of each fly to different inactivity bins also revealed these to be evenly distributed across individuals (Fig. 5C,D). For example, any fly that had provided responsiveness data for $20-40 \mathrm{~min}$ inactivity bins also provided responsiveness data for $0-20 \mathrm{~min}$ inactivity bins (i.e., these were not different groups of flies). However, it is clear that all flies contributed less to 20-40 min inactivity bins compared with 0-20 min and 40-60 min inactivity bins (Fig. 5 E, F). One explanation for this distribution of the data could be that sleep intensity levels were indeed cycling within an extended sleep bout: there may have been fewer 20-40 min events because flies that have slept through the end of their first sleep cycle will most likely initiate the next sleep cycle and therefore not wake up during the deeper stages (i.e., centered around $30 \mathrm{~min}$ ) of their second sleep cycle. Most flies, however, would naturally awaken after $20 \mathrm{~min}$, which would be consistent with our averaged data showing that sleeping flies are comparatively more responsive at that time (Fig. $4 C, D)$ and with the average sleep bout length being $\sim 20-30 \mathrm{~min}$ in our wild-type strain (see Materials and Methods). We therefore focused on the first $20 \mathrm{~min}$ of sleep, which includes the first deep sleep stage, for the remainder of this study.

To better identify the first deep sleep stage, we fit a third-order polynomial to smoothed responsiveness data (see Materials and Methods), with the first local minimum in the curve indicating the time of deepest sleep (Fig. 6A, $B$, vertical dashed lines). The first deep sleep stage was found to occur between 12 and 15 min of inactivity in wild-type flies regardless of time of day or sex, which suggests strongly regulated processes controlling sleep intensity in Drosophila. When we tested for responsiveness every $30 \mathrm{~min}$
A

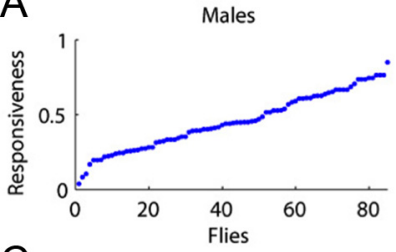

C
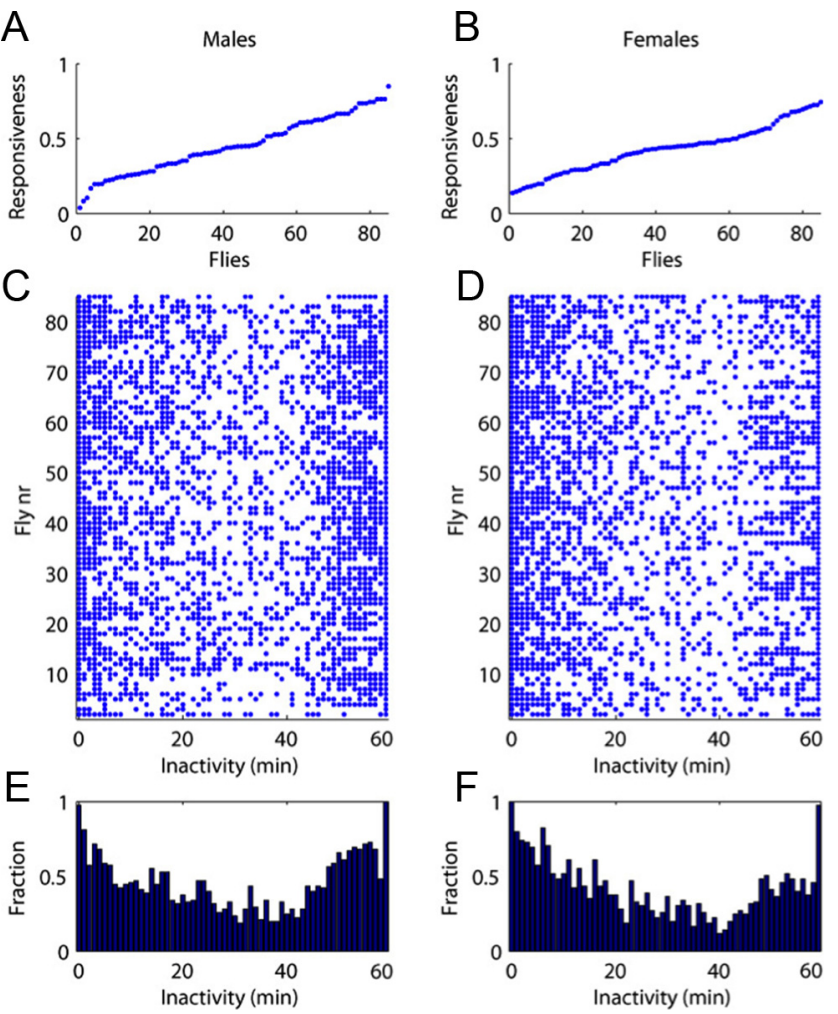

Figure 5. Individual fly sleep data distribution. $A, B$, Distribution of average responsiveness levels (response probability) for wild-type male $(n=85)$ and female $(n=85)$ flies discussed in Figure $4 C$, $D$ sorted from lowest to highest responders (only flies that were inactive before the startle test are represented). Responsiveness is normally distributed among flies (Lilliefors test, $p<0.05$ ), indicating that the different sleep intensity levels after sleep onset (Fig. $4 C, D$ ) are unlikely to reflect subpopulations of flies behaving differently. $\boldsymbol{C}, \boldsymbol{D}$, Distribution of inactivity data for each fly (same flies as in $\boldsymbol{A}, \boldsymbol{B}$ ). The blue dots show, for each fly, whether it contributed to each inactivity bin ( $0-59 \mathrm{~min})$. $(\boldsymbol{E}, \boldsymbol{F})$ By summing data from $\boldsymbol{C}$ and $\boldsymbol{D}$, we determined the proportion (fraction) of flies contributing to each bin. Fewer flies contribute to middle-length inactivity bins (20-40 min), although data within this epoch are evenly distributed among flies.

instead of every hour in a replicate set of experiments, the first deep sleep stage seemed earlier and deeper, especially in males (Fig. 6C,D), suggesting that a 30 min testing regime may be increasing sleep drive (i.e., producing some sleep deprivation). Similarly, our brain-recording paradigm yielded a comparatively earlier onset of lower $10-40 \mathrm{~Hz}$ activity (Fig. 2E), suggesting that the tethered recording setup may have caused some sleep deprivation. We therefore focused on an hourly sleep probe in subsequent experiments.

\section{Behavioral effects on sleep intensity}

We next examined the effect of sleep drive on sleep intensity dynamics. In humans, SWS duration and intensity increase proportionally to prior wake time and decrease as sleep is restored throughout the night (Borbély and Achermann, 1999), evidence that sleep is a homeostatic process. Some of our data already suggest a homeostatic process: quiescent male flies were less responsive in the first hours of the night compared with equally quiescent flies in the last hours of the night (Fig. 4B). As in humans, extended wakefulness in Drosophila males and females results in increased sleep drive (pressure to sleep) (Hendricks et al., 2000; Shaw et al., 2000). To determine more directly whether increased wakefulness alters sleep intensity profiles, we sleep deprived flies for $24 \mathrm{~h}$ (see Materials and Methods) and measured 


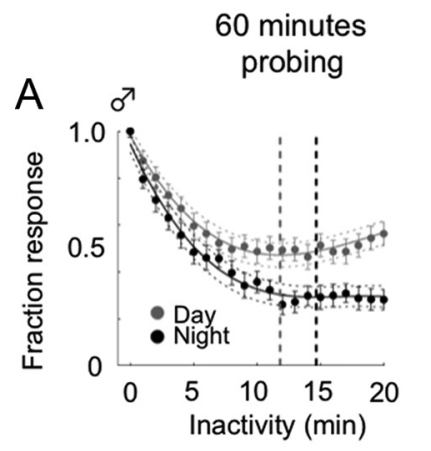

30 minutes probing

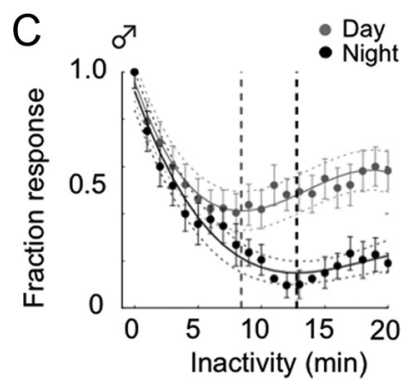

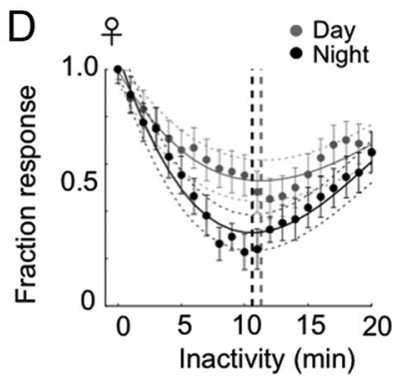

Figure 6. The first deep-sleep stage. $\boldsymbol{A}, \boldsymbol{B}$, To quantify deep sleep intensity and timing, third-order polynomials were fitted to smoothed mean responsiveness data from Fig. $4 C, D: A$, males; $\boldsymbol{B}$, females, $n=85$ in both groups) for the first 20 min of inactivity (see Materials and Methods). The local minimum in the curve indicates the first deep sleep stage (vertical dashed lines at $\sim 12-15 \mathrm{~min})$. Curves show polynomials fitted to means \pm SEM. $A$, Day, $r^{2}=0.97$; night, $r^{2}=0.98 . \boldsymbol{B}$, Day, $r^{2}=0.96 ;$ night, $r^{2}=0.98 . C, D, A 1.2$ g vibration stimulus was used to probe responsiveness every 30 min over three consecutive days in separate group of 85 wild-type males and females. Gray circles indicate mean daytime responsiveness; black circles, mean nighttime responsiveness ( \pm SEM).

sleep intensity for the following day or night. We found that $24 \mathrm{~h}$ of sleep deprivation precipitated the onset of deep sleep in males and females (Fig. $7 A, B$, vertical dashed lines). To quantify sleep intensity at this time, we averaged the (non-smoothed) data for a 5 min window around the point of deepest sleep (Fig. 7C), termed the deep sleep response probability. Deep sleep response probability was significantly lower in sleep-deprived flies, but only for nighttime sleep (Fig. 7D). Importantly, sleep intensity returned to comparatively lighter levels after a full day of recovery for both day and night in males (Fig. 7E, one-way ANOVA $F_{(1,135)}=5.42$, $p=0.02$; and $F_{(1,135)}=11.78, p<0.001$, respectively). Female recovery from sleep deprivation was less clear; only daytime responsiveness appeared to recover on the second day (Fig. $7 F$, one-way ANOVA $\left.F_{(1,135)}=11.14, p=0.001\right)$.

Other behavioral manipulations predicted to increase sleep intensity are socialization and learning. The synaptic homeostasis hypothesis for sleep (Tononi and Cirelli, 2006) describes an interaction between wake experience and sleep drive: information encoded during wake leads to an overall increase in synaptic strength in the brain, which would then be proportionally normalized (i.e., downscaled) during the deeper stages of sleep. Because socialization has been proposed as one way to increase sleep drive in Drosophila (Ganguly-Fitzgerald et al., 2006), we tested the effect of this simple manipulation on sleep intensity in our arousal paradigm. Socialized male flies (males kept with other males for $4 \mathrm{~d}$; see Materials and Methods) slept more deeply subsequently during the day compared with similarly handled male flies kept in isolation (Fig. $8 A, B$ ). In contrast, sleep intensity
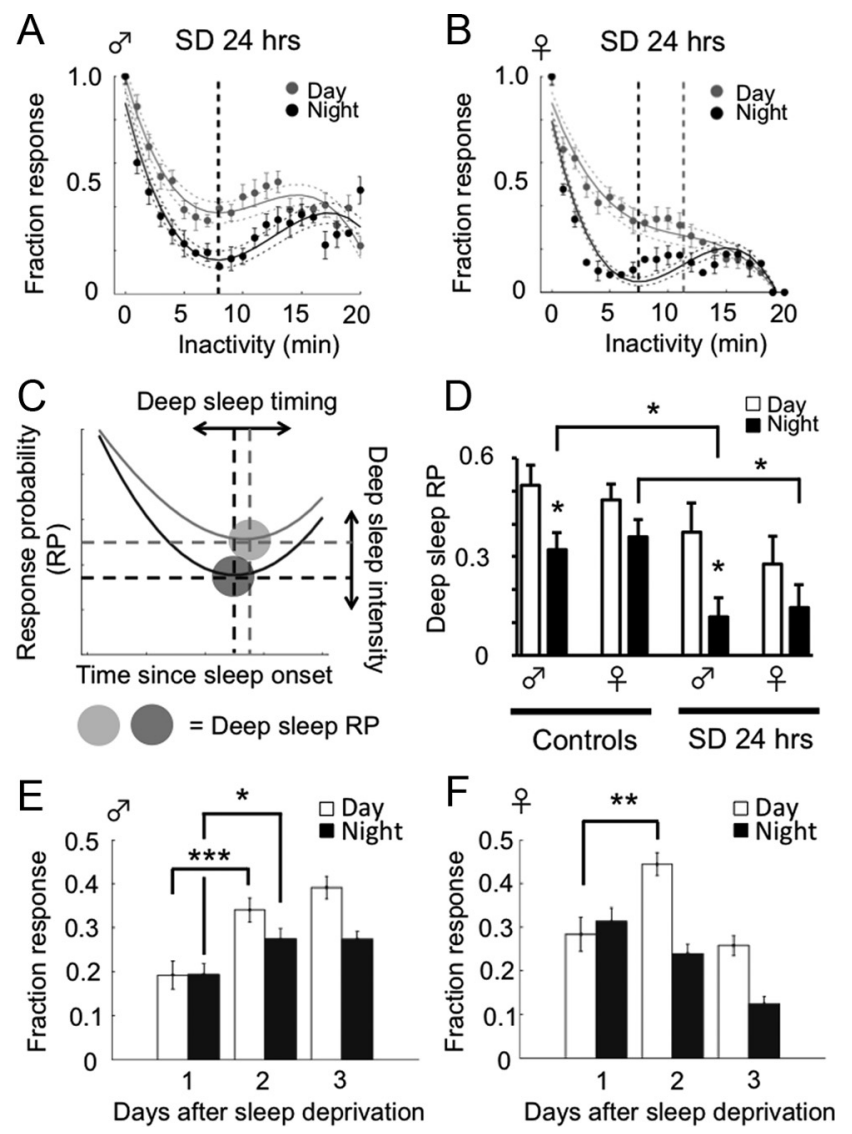

Figure 7. Effect of sleep deprivation on the first deep sleep stage. $\boldsymbol{A}, \boldsymbol{B}$, After $24 \mathrm{~h}$ of sleep deprivation (SD), sleep initiation occurs faster and sleep is deeper when recovery sleep occurs during the day or the night ( $\boldsymbol{A}$, males; $\boldsymbol{B}$, females, $n=68$ in both groups). $\boldsymbol{A}$, Day, $r^{2}=0.92$; night, $r^{2}=0.84$. $B$, Day, $r^{2}=0.96 ;$ night, $r^{2}=0.89$. C, Schema showing how deepest sleep timing is identified, with corresponding response probability (RP) at that time. Deep sleep RP was determined by averaging (non-smoothed) responsiveness data for a 5 min epoch centered around the deepest sleep time. $\boldsymbol{D}$, Deep sleep RP averages ( \pm SEM) for data from $\boldsymbol{A}$ and $\boldsymbol{B}$ comparing daytime and nighttime sleep intensity and SD and non-SD controls. ${ }^{*} p<0.05$ by Wilcoxon rank-sum test. $\boldsymbol{E}$, Average responsiveness during day and night for three consecutive days after $24 \mathrm{~h}$ of sleep deprivation in wild-type males $(n=68)$. A significant increase in responsiveness can be observed between days 1 and 2 after sleep deprivation, suggesting that decreased responsiveness on day 1 was due to deeper rebound sleep and not to damage caused by the sleep deprivation procedure. ${ }^{*} p<0.05, F_{(1,135)}=5.42 ;{ }^{* *} p<0.001$. $\boldsymbol{F}$, Average responsiveness during day and night for three consecutive days after $24 \mathrm{~h}$ of sleep deprivation in wild-type females $(n=68)$. ${ }^{* *} p<0.01$.

in female flies was not affected by intrasexual socialization (Fig. $8 C, D$ ), even though sleep duration was altered (Fig. $8 E, F$ ), as measured by more traditional (i.e., infrared beam crossing) sleep metrics. This result highlights an important point: longer sleep duration does not necessarily mean that the flies were sleeping more deeply. The dimorphism in sleep intensity between males and females may be due to different behavioral interactions among males or females, with more complex male social interactions such as aggression and courtship producing a greater sleep drive during the day. These social interactions may, for example, engage learning and memory pathways that affect sleep intensity in males.

\section{Optogenetic manipulation of sleep intensity}

To investigate a possible link between learning and sleep intensity in flies (Seugnet et al., 2008), we manipulated acutely a molecular pathway that has been associated with synaptic plasticity and memory formation. Learning and memory processes are closely 

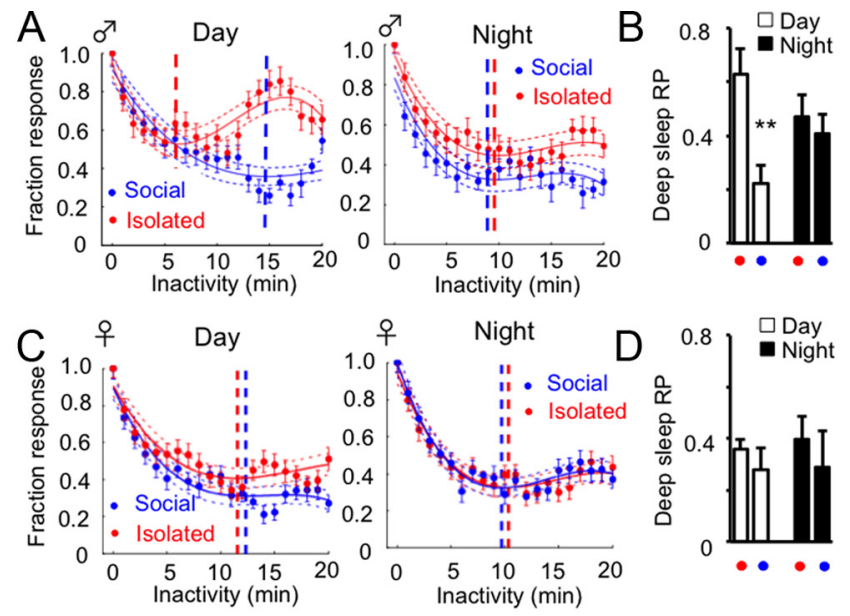

E
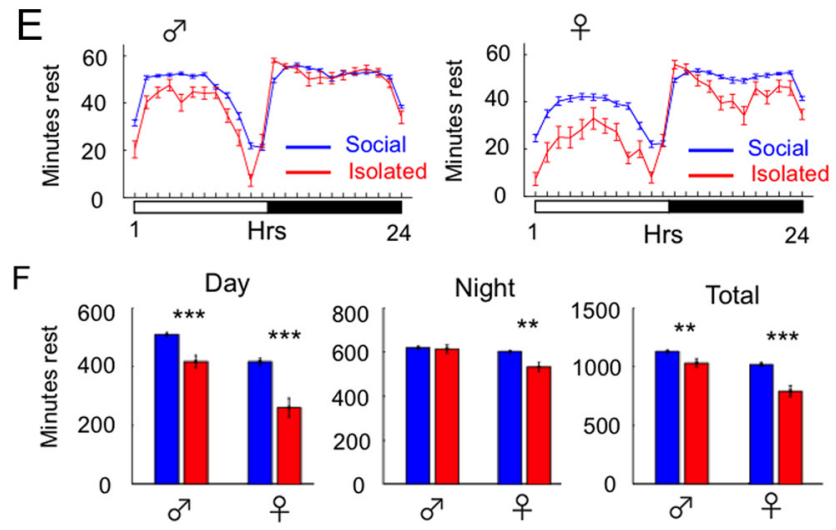

Figure 8. Effect of socialization on fly sleep intensity. $A, B$, Socialization increases deep sleep response probability (RP; \pm SEM) in males during the day (blue, $n=51$ ) compared with males kept in isolation (red, $n=51$ ). ${ }^{* *} p<0.05$ by Wilcoxon rank-sum test. The vertical lines indicate the point of deepest sleep, from which deep sleep RP was calculated (see Materials and Methods). C, D, Socialization does not significantly change sleep intensity (deep sleep RP \pm SEM) in females, day or night (blue, $n=51$ ), compared with females kept in isolation (red, $n=$ 51). $\boldsymbol{E}$, Activity was measured using the DAMS (see Materials and Methods). Rest is defined as 5 min or longer without any detected activity in the DAMS, cumulative for every hour ( \pm SEM, $n=32$ flies per sex or condition). Socially reared adult flies (blue traces) are compared with 5 to 6-d-old flies that were isolated in plastic vials soon after eclosion (red traces) under the same conditions as in $\boldsymbol{A}-\boldsymbol{D}$. Flies are wild-type CS. $\boldsymbol{F}$, Daytime and total sleep duration ( \pm SEM) is decreased in both male and female flies that have been raised in isolation (red; socially reared flies are blue) and tracked by the DAMS system. Data are processed from flies in $\boldsymbol{E} .{ }^{* * *} p<$ $0.001 ;{ }^{* *} p<0.01$ by Wilcoxon rank-sum test.

tied to cyclic adenosine monophosphate (cAMP) signaling pathways in neurons, where increased cAMP activity results in increased synaptic efficacy (Huang et al., 1994) and in the transcription of genes involved in upregulating synaptic strength (Dash et al., 1990). Fly memory mutants defective in cAMP regulation, such as dunce ${ }^{1}$ (which has chronically increased cAMP levels), sleep less (Hendricks et al., 2001) and do not show experience-dependent changes in sleep duration (GangulyFitzgerald et al., 2006). We therefore sought to specifically increase cAMP activity only during the day, when most learning behavior presumably occurs, to determine whether acute molecular control of plasticity mechanisms in awake flies altered sleep intensity. To best achieve this, we used an optogenetic approach: a transgenic adenylyl cyclase that is activated by blue light, $P A C \alpha$ (Schröder-Lang et al., 2007), under control of the UAS/Gal4 expression system (Brand and Perrimon, 1993). Under blue light, PAC $\alpha$ converts ATP into cAMP in Gal4-specified neurons (Fig. $9 A$ ), initiating a signaling cascade that modulates synaptic func-
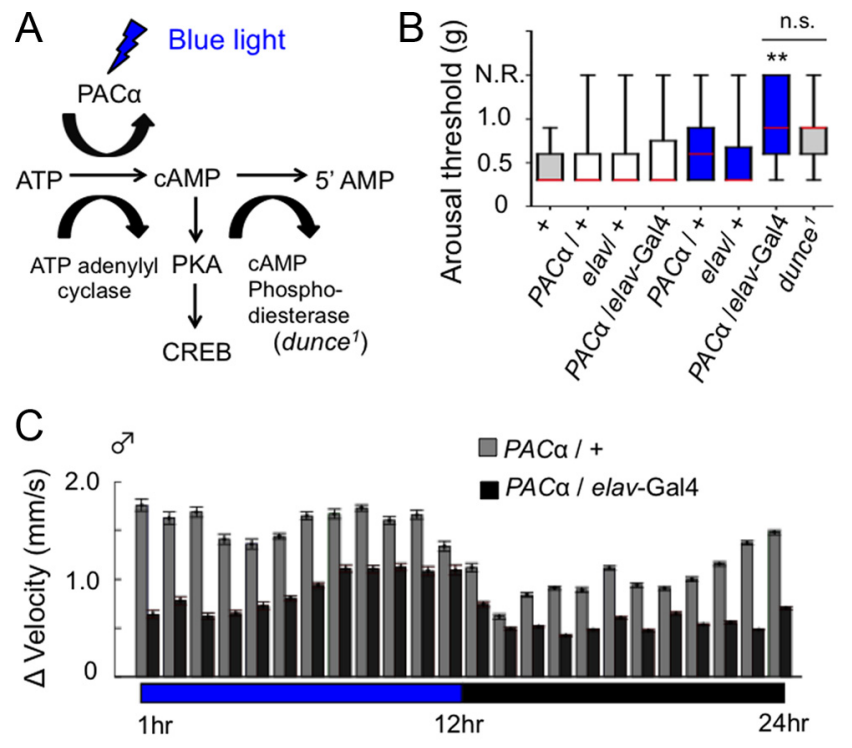

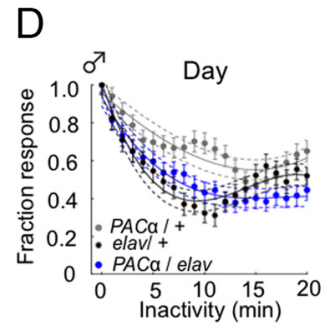

$\mathrm{E}$

Figure 9. Adenylyl cyclase activity increases nighttime sleep intensity. A, cAMP synthesis and degradation pathway. A transgenic adenylyl cyclase $(P A C \alpha)$ is activated by blue light, resulting in increased levels of cAMP and consequent plasticity signaling mechanisms (PKA and (REB). Increased CAMP levels are also achieved by the phosphodiesterase gene mutation, dunce ${ }^{1} . \boldsymbol{B}$, Acute blue light activation of $P A C \alpha$ increases arousal thresholds to levels similar to dunce ${ }^{1}$ mutants. Arousal thresholds (the minimal vibration, $g$, required to arouse a quiescent fly; see Materials and Methods) for wild-type (,$+ n=42)$, UAS-PAC $\alpha /+(n=36)$, elavGal4/+ $(n=34)$, UAS-PAC $\alpha /$ elav-Gal4 $(n=37)$, and dunce $e^{1}(n=71)$. White boxplots indicate white light exposure; blue boxplots, 10 min blue light exposure. ${ }^{* *} p<0.01$ by KruskalWallis comparison of medians (red) adjusted for multiple comparisons. C, Baseline and stimulus-induced velocity ( \pm SEM) for UAS-PAC $\alpha /+$ control male flies and UAS-PAC $\alpha /$ elavGal4 male flies in response to hourly stimulation by $1.2 \mathrm{~g}$ for $72 \mathrm{~h}$ averaged over a $24 \mathrm{~h}$ period. Blue light $(458 \pm 10 \mathrm{~nm})$ was turned on throughout the day and turned off at night for both. $D$, $E$, Acute activation of $P A C \alpha$ with blue light during the day increases sleep intensity at night in males. The sleep intensity profiles for UAS-PAC $\alpha /+$ (gray, $n=85)$, elav-Gal $4 /+($ black, $n=$ 85 ), and the blue-light-activated UAS-PAC $\alpha /$ elav-Gal4 (blue, $n=85$ ) is shown ( \pm SEM). Third-order polynomials were fitted to normalized, smoothed mean responsiveness data for the first 20 min of inactivity for daytime or nighttime sleep. Day: UAS-PAC $\alpha /+, r^{2}=0.85$; elavGal4/,$+ r^{2}=0.84$; UAS-PAC $\alpha /$ elav-Gal4, $r^{2}=0.97$. Night: UAS-PAC $\alpha /+, r^{2}=0.88$; elavGal4/+, $r^{2}=0.77$; UAS-PAC $\alpha /$ elav-Gal4, $r^{2}=0.94$. $F$, Deep sleep response probability (RP) data and statistics. ${ }^{*} p<0.05$ by Wilcoxon rank-sum test.

tion (Huang et al., 1994; Schröder-Lang et al., 2007). We found that acute, pan-neuronal activation of $P A C \alpha$ (in elav-Gal4/UAS$P A C \alpha$ flies) significantly increased arousal thresholds (decreased responsiveness) to levels similar to that in dunce ${ }^{1}$ mutants (Fig. $9 B, p<0.01$, Kruskal-Wallis test comparing medians, $3 \mathrm{df}, \mathrm{H}=$ 12.63). When adenylyl cyclase activation was continued for the flies' entire day (but turned off at night), elav-Gal4/UAS-PAC $\alpha$ flies displayed chronically decreased responsiveness to mechanical stimuli during both day and night compared with controls also exposed to blue light (Fig. 9C). However, sleep intensity was only significantly increased at night, after blue light was turned off, compared with both genetic controls (Fig. 9D-F). This sug- 
A

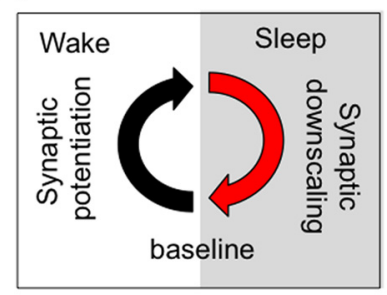

B
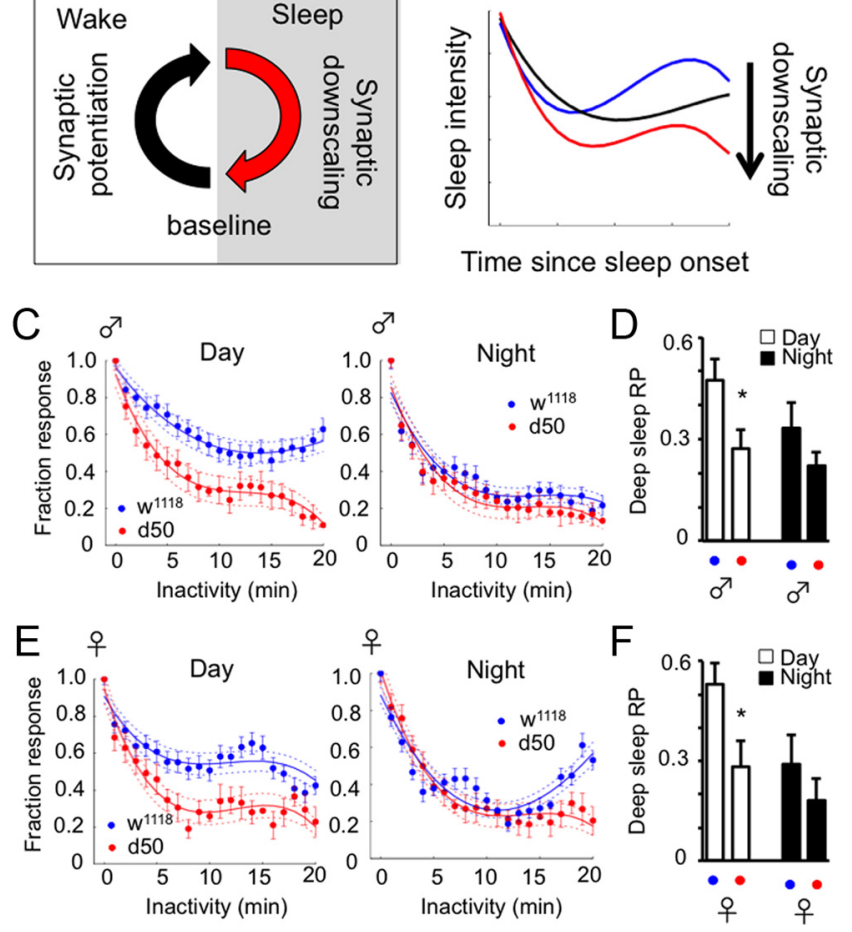

Figure 10. Loss of $d F m r 1$ gene function increases daytime sleep intensity. $\boldsymbol{A}$, Synaptic homeostasis hypothesis schema. $\boldsymbol{B}$, Synaptic downscaling is hypothesized to be engaged by increased sleep intensity. $\boldsymbol{C}$, Male sleep intensity profiles for up to 20 min of inactivity during the light and dark period based on $1 \mathrm{~min}$ bins for a loss of function mutant of the fragile-X mental retardation gene ( $\mathrm{d} 50, n=34$, red) compared with its genetic background control ( $\mathrm{w}^{1118}, n=$ 68, blue). Day: $w^{1118} r^{2}=0.91 ; d 50, r^{2}=0.96$. Night: $w^{1118} r^{2}=0.80 ; d 50, r^{2}=0.95$. D, Deep sleep response probability (RP) and statistics, data from $C$. ${ }^{*} p<0.05$ by Wilcoxon ranksum test. $\boldsymbol{E}$, Female sleep intensity profiles for day and night for a loss of function mutant of the Fmr1 gene (d50, $n=34$, red graph, compared with its genetic background control ( $\mathrm{w}^{1118}, n=$ 68 , blue graph). Data are normalized means fit by a third-order polynomial (see Materials and Methods). $\mathrm{w}^{1118} /+, r^{2}=0.68 ; \mathrm{d} 50, r^{2}=0.95 . \mathrm{w}^{1118} /+, r^{2}=0.80 ; \mathrm{d} 50, r^{2}=0.98$. F, Deep sleep RP data and statistics for sleep intensity data in $\boldsymbol{E}$. ${ }^{*} p<0.05$ by Wilcoxon rank-sum test.

gests that cAMP-related signaling that accrues during the day has long-lasting effects on sleep intensity at night.

\section{Synaptic homeostasis and sleep intensity}

The synaptic homeostasis hypothesis for sleep proposes that synaptic potentiation accrued during the day is proportionally downscaled during sleep (Tononi and Cirelli, 2003, 2006; Fig. $10 A$ ). A protein closely associated with synaptic remodeling and sleep function is FMRP, produced by the fragile-X mental retardation gene $(F m r 1)$, which regulates synaptic pruning (Tessier and Broadie, 2008) and synaptic plasticity (Mercaldo et al., 2009). Sleep duration in flies is tightly regulated by $\mathrm{dFmr1}$, the Drosophila homolog of the gene (Wan et al., 2000). In Drosophila, loss of $d F m r 1$ results in overgrown dendrites (Pan et al., 2004; Bushey et al., 2011), whereas $d F m r 1$ overexpression has the opposite effects: reduced dendritic branching and loss of synaptic differentiation (Pan et al., 2004). Increased sleep duration in dFmr1 loss of function mutants is therefore thought to result from less efficient synaptic downscaling during sleep, although an association with deeper sleep, predicted in humans (Tononi and Cirelli, 2006), has never been shown in flies. Our results thus far suggest that deeper sleep may be associated with homeostatic functions in the fly brain (Fig. 10B). What would be the effect on sleep intensity if a component of the synaptic downscaling machinery were defec- tive? Using our arousal paradigm to test $d F m r 1$ mutants, we found that a loss-of-function allele of $d F m r 1$ (d50) displayed deeper night-like sleep during the day (Fig. 10C,D). Female dFmr1 mutants showed a similar significant effect (Fig. 10E,F). Therefore, rather than further deepening nighttime sleep (as in the case of sleep-deprived flies; Fig. 7D), loss-of-function of dFmr1 mainly deepened daytime sleep.

\section{Discussion}

The discovery over a decade ago that $D$. melanogaster sleeps (Hendricks et al., 2000; Shaw et al., 2000) has revolutionized approaches to studying sleep functions in animal models. This is because the powerful tools associated with Drosophila genetic analysis could be applied to understanding sleep functions, which had traditionally been studied in higher animals less amenable to molecular genetic analysis. Increasingly, Drosophila sleep phenotypes are now being used as a model to study the molecular underpinnings of cognitive disorders such as schizophrenia and mental retardation (van Alphen and van Swinderen, 2013), with the idea that a fundamental connection is likely to exist between many brain disorders and defects in sleep functions. One of the proposed functions of sleep that may provide this connection is synaptic homeostasis (Tononi and Cirelli, 2006). According to this hypothesis (which remains actively debated; Frank, 2012; Tononi and Cirelli, 2012), deep sleep in mammals serves to downscale synaptic strengths proportionally across the brain, thereby decreasing energy and space requirements while preserving the relative synaptic weights that are a manifestation of learning and plasticity. Indeed, some cellular and molecular evidence for synaptic downscaling during sleep has come from recent research in Drosophila (Donlea et al., 2009; Gilestro et al., 2009; Bushey et al., 2011). However, one setback with using Drosophila to study this mammal-centric hypothesis is that deep sleep specifically has been proposed as the stage during which synaptic downscaling occurs, with delta $(0.5-4 \mathrm{~Hz})$ "slow" waves proposed as the mechanism that may enable downscaling (Tononi and Cirelli, 2006). However, despite earlier work suggesting simple approaches to identifying sleep intensity in flies (Huber et al., 2004; Andretic and Shaw, 2005), most Drosophila sleep studies often equate total sleep duration with sleep intensity: the longer flies are inactive (as determined by infrared beam crossing devices), the more sleep functions are presumably being accomplished. Our study shows that sleep duration does not necessarily equate with sleep intensity: flies can sleep more lightly or more deeply at different times of the day or times since sleep onset. In addition, there is no strong evidence so far of slow-wave activity during sleep in flies or other insects (van Swinderen, 2006). Therefore, the use of the powerful Drosophila model to investigate sleep functions originally proposed for mammals may have met with some resistance because there was no evidence of different sleep stages in flies and no evidence of distinct electrical signatures in the fly brain associated with sleep intensity, two key sleep criteria in mammals. Our study identifies a deep sleep stage in flies.

A synthesis of our electrophysiological, behavioral, and genetic manipulations demonstrates that sleep in flies, as in mammals and birds, is a dynamic, heterogeneous state, which suggests that different sleep stages are a fundamental characteristic of sleep in any animal. Sleep in Drosophila transitions through stereotypical epochs of increasing and decreasing intensity, and our results show that behavioral and genetic manipulations can alter the timing and intensity of the first deep sleep stage. Importantly, different strains may display different baseline sleep intensity 
profiles (e.g., baseline sleep appears lighter in Pac $\alpha /+$ controls; Fig. $9 D, E)$, so a 5 min threshold for defining sleep in Drosophila may not be appropriate for all strains. Furthermore, daytime sleep is lighter on average than nighttime sleep, so accumulating sleep duration metrics by combining daytime and nighttime sleep (e.g., "total sleep") may not always be valid. Finally, sleep intensity can become lighter as flies remain inactive, so total length of a sleep bout does not necessarily reveal what kind of sleep is occurring at any one time. This may especially be the case for mutant strains, in which sleep duration may not reflect normal sleep functions. We find, for example, that $d F m r 1$ mutants sleep more deeply during the day, whereas nighttime sleep intensity remains unchanged. This suggests that loss of FMRP-related synaptic downscaling transfers nighttime sleep functions to the day, perhaps to offset the less efficient downscaling that occurs during the night in the mutant. Conversely, cAMP upregulation during the day deepens nighttime sleep, perhaps because more downscaling is required after this artificial upregulation of synaptic activity. Finally, our socialization protocol appeared to only increase daytime sleep intensity and only in males.

The existence of a deep sleep stage in Drosophila raises the question of why flies also display a substantial amount of lighter sleep, especially during the day. Indeed, the nonequivalence of daytime and nighttime sleep was observed in most of our experiments and manipulations, supporting the idea that daytime and nighttime sleep achieve distinct functions in flies (Ishimoto et al., 2012), which we propose can also be understood as lighter and deeper sleep stages. If the function of deep sleep in flies and other animals is associated with synaptic downscaling, why is there also a need for extensive periods of light sleep? Are the behavioral defects in $\mathrm{AFmr} 1$ mutants due to defective deep sleep processes or to lost light sleep processes? Our finding that sleep intensity is regulated by two molecules (FMRP and cAMP) involved in plasticity and synaptic remodeling allows us to speculate that a distinct suite of molecules is expressed to achieve specific sleep functions in correlation with the timing and depth of the deep sleep stage (Fig. 10B). By considering how sleep intensity and timing changes after behavioral manipulations or in mutant strains, future studies should uncover precise, functional roles for sleep processes in the Drosophila model. Although flies do not appear to display the "delta" slow waves (Nitz et al., 2002) that have been proposed as a mechanism for synaptic downscaling during mammalian deep sleep (Tononi and Cirelli, 2006), it is possible that reduced oscillatory activity within behaviorally relevant frequency ranges (e.g., 11-40 Hz) may accomplish similar synaptic downscaling functions in simpler animals with smaller brains. Although we have not combined electrophysiology with arousal threshold experiments in this study, the first deep sleep stage in Drosophila appears to be matched by transiently floored 11-40 Hz LFP activity in the brain. Because increased activity in a similar LFP frequency range has been associated with selective attention and choice behavior in awake flies (van Swinderen and Greenspan, 2003; Tang and Juusola, 2010), it is possible that transiently decreased activity in this same frequency range reflects a homeostatic downscaling response during deep sleep in insects. Future studies should establish the connection, if any, between global changes in electrical activity in the fly brain and expression of genes involved in regulating synaptic function.

\section{References}

Andretic R, Shaw PJ (2005) Essentials of sleep recordings in Drosophila: moving beyond sleep time. Methods Enzymol 393:759-772. CrossRef Medline
Blake H, Gerard R (1937) Brain potentials during sleep. Am J Physiol 119:692-703.

Borbély AA, Achermann P (1999) Sleep homeostasis and models of sleep regulation. J Biol Rhythms 14:557-568. Medline

Brand AH, Perrimon N (1993) Targeted gene expression as a means of altering cell fates and generating dominant phenotypes. Development 118: 401-415. Medline

Bushey D, Tononi G, Cirelli C (2009) The Drosophila fragile X mental retardation gene regulates sleep need. J Neurosci 29:1948-1961. CrossRef Medline

Bushey D, Tononi G, Cirelli C (2011) Sleep and synaptic homeostasis: structural evidence in Drosophila. Science 332:1576-1581. CrossRef Medline

Campbell SS, Tobler I (1984) Animal sleep: a review of sleep duration across phylogeny. Neurosci Biobehav Rev 8:269-300. CrossRef Medline

Cirelli C, Tononi G (2008) Is sleep essential? PLoS Biol 6:e216. CrossRef Medline

Dash PK, Hochner B, Kandel ER (1990) Injection of the cAMP-responsive element into the nucleus of Aplysia sensory neurons blocks long-term facilitation. Nature 345:718-721. CrossRef Medline

Diekelmann S, Born J (2010) The memory function of sleep. Nat Rev Neurosci 11:114-126. CrossRef Medline

Donlea JM, Ramanan N, Shaw PJ (2009) Use-dependent plasticity in clock neurons regulates sleep need in Drosophila. Science 324:105-108. CrossRef Medline

Eban-Rothschild AD, Bloch G (2008) Differences in the sleep architecture of forager and young honeybees (Apis mellifera). J Exp Biol 211:24082416. CrossRef Medline

Frank MG (2012) Erasing synapses in sleep: is it time to be SHY? Neural Plast 2012:264378. CrossRef Medline

Ganguly-Fitzgerald I, Donlea J, Shaw PJ (2006) Waking experience affects sleep need in Drosophila. Science 313:1775-1781. CrossRef Medline

Gilestro GF, Cirelli C (2009) pySolo: a complete suite for sleep analysis in Drosophila. Bioinformatics 25:1466-1467. CrossRef Medline

Gilestro GF, Tononi G, Cirelli C (2009) Widespread changes in synaptic markers as a function of sleep and wakefulness in Drosophila. Science 324:109-112. CrossRef Medline

Hendricks JC, Finn SM, Panckeri KA, Chavkin J, Williams JA, Sehgal A, Pack AI (2000) Rest in Drosophila is a sleep-like state. Neuron 25:129-138. CrossRef Medline

Hendricks JC, Williams JA, Panckeri K, Kirk D, Tello M, Yin JC, Sehgal A (2001) A non-circadian role for cAMP signaling and CREB activity in Drosophila rest homeostasis. Nat Neurosci 4:1108-1115. CrossRef Medline

Huang YY, Li XC, Kandel ER (1994) cAMP contributes to mossy fiber LTP by initiating both a covalently mediated early phase and macromolecular synthesis-dependent late phase. Cell 79:69-79. CrossRef Medline

Huber R, Hill SL, Holladay C, Biesiadecki M, Tononi G, Cirelli C (2004) Sleep homeostasis in Drosophila melanogaster. Sleep 27:628-639. Medline

Ishimoto H, Lark A, Kitamoto T (2012) Factors that differentially affect daytime and nighttime sleep in Drosophila melanogaster. Front Neurol 3:24. CrossRef Medline

Killgore WD (2010) Effects of sleep deprivation on cognition. Prog Brain Res 185:105-129. CrossRef Medline

Mercaldo V, Descalzi G, Zhuo M (2009) Fragile X mental retardation protein in learning-related synaptic plasticity. Mol Cells 28:501-507. CrossRef Medline

Nitz DA, van Swinderen B, Tononi G, Greenspan RJ (2002) Electrophysiological correlates of rest and activity in Drosophila melanogaster. Curr Biol 12:1934-1940. CrossRef Medline

Pan L, Zhang YQ, Woodruff E, Broadie K (2004) The Drosophila fragile X gene negatively regulates neuronal elaboration and synaptic differentiation. Curr Biol 14:1863-1870. CrossRef Medline

Ramón F, Hernández-Falcón J, Nguyen B, Bullock TH (2004) Slow wave sleep in crayfish. Proc Natl Acad Sci U S A 101:11857-11861. CrossRef Medline

Rechtschaffen A, Kales A (1968) A manual of standardized terminology, techniques and scoring system of sleep stages in human subjects. Los Angeles: Brain Information Service/Brain Research Institute.

Rechtschaffen A, Hauri P, Zeitlin M (1966) Auditory awakening thresholds in REM and NREM sleep stages. Percept Mot Skills 22:927-942. CrossRef Medline 
Sauer S, Kinkelin M, Herrmann E, Kaiser W (2003) The dynamics of sleeplike behaviour in honey bees. J Comp Physiol A Neuroethol Sens Neural Behav Physiol 189:599-607. CrossRef Medline

Sawamura N, Ando T, Maruyama Y, Fujimuro M, Mochizuki H, Honjo K, Shimoda M, Toda H, Sawamura-Yamamoto T, Makuch LA, Hayashi A, Ishizuka K, Cascella NG, Kamiya A, Ishida N, Tomoda T, Hai T, Furukubo-Tokunaga K, Sawa A (2008) Nuclear DISC1 regulates CREmediated gene transcription and sleep homeostasis in the fruit fly. Mol Psychiatry 13:1138-1148, 1069. CrossRef Medline

Schröder-Lang S, Schwärzel M, Seifert R, Strünker T, Kateriya S, Looser J, Watanabe M, Kaupp UB, Hegemann P, Nagel G (2007) Fast manipulation of cellular cAMP level by light in vivo. Nat Methods 4:39-42. CrossRef Medline

Seugnet L, Suzuki Y, Vine L, Gottschalk L, Shaw PJ (2008) D1 receptor activation in the mushroom bodies rescues sleep-loss-induced learning impairments in Drosophila. Curr Biol 18:1110-1117. CrossRef Medline

Shaw PJ, Cirelli C, Greenspan RJ, Tononi G (2000) Correlates of sleep and waking in Drosophila melanogaster. Science 287:1834-1837. CrossRef Medline

Shaw PJ, Tononi G, Greenspan RJ, Robinson DF (2002) Stress response genes protect against lethal effects of sleep deprivation in Drosophila. Nature 417:287-291. CrossRef Medline

Tang S, Juusola M (2010) Intrinsic activity in the fly brain gates visual information during behavioral choices. PLoS One 5:e14455. CrossRef Medline

Tessier CR, Broadie K (2008) Drosophila fragile X mental retardation pro- tein developmentally regulates activity-dependent axon pruning. Development 135:1547-1557. CrossRef Medline

Tobler I I, Neuner-Jehle M (1992) 24-h variation of vigilance in the cockroach Blaberus giganteus. J Sleep Res 1:231-239. CrossRef Medline

Tononi G, Cirelli C (2003) Sleep and synaptic homeostasis: a hypothesis. Brain Res Bulletin 62:143-150. CrossRef Medline

Tononi G, Cirelli C (2006) Sleep function and synaptic homeostasis. Sleep Med Rev 10:49-62. CrossRef Medline

Tononi G, Cirelli C (2012) Time to be SHY?-some comments on sleep and synaptic homeostasis. Neural Plast 2012:415250. CrossRef Medline

van Alphen B, van Swinderen B (2013) Drosophila strategies to study psychiatric disorders. Brain Res Bull 92:1-11.

van Swinderen B (2012) Competing visual flicker reveals attention-like rivalry in the fly brain. Front Integr Neurosci 6:96. CrossRef Medline

van Swinderen B, Greenspan RJ (2003) Salience modulates 20-30 Hz brain activity in Drosophila. Nat Neurosci 6:579-586. CrossRef Medline

van Swinderen B, Nitz DA, Greenspan RJ (2004) Uncoupling of brain activity from movement defines arousal states in Drosophila. Curr Biol 14:8187. CrossRef Medline

van Swinderen (2006) Sleep in invertebrates. In: Evolution of nervous systems (Kaas JH, ed). Oxford: Academic.

Wan L, Dockendorff TC, Jongens TA, Dreyfuss G (2000) Characterization of dFMR1, a Drosophila melanogaster homolog of the fragile X mental retardation protein. Mol Cell Biol 20:8536-8547. CrossRef Medline

Webb WB, Agnew HW Jr (1971) Stage 4 sleep: influence of time course variables. Science 174:1354-1356. CrossRef Medline 